\title{
Classic Spotlight, 2010 and 2011: Articles of Significant Interest Selected from the Journal of Virology Archives by the Editors
}

\begin{abstract}
Ournal of Virology (JVI) marks its 50th year of publishing in 2017. To highlight particularly noteworthy $\mathrm{JVI}$ articles from over the years, 2017 issues are featuring Classic Spotlights selected from the archives by the editors. These Classic Spotlights are appearing chronologically, and in this issue, we have selected articles from 2010 and 2011.
\end{abstract}

Regulation of Hepatitis C Virus Translation and Infectious Virus Production by the MicroRNA miR-122

A liver-specific microRNA, miR-122, positively regulates hepatitis $C$ virus (HCV) RNA abundance and is essential for production of infectious HCV. Jangra et al. (R. K. Jangra, M. Yi, and S. M. Lemon, J Virol 84:6615-6625, 2010, https://doi.org/10.1128/JVI.00417 -10) used a genetic approach to show that a higher yield of infectious virus was dependent upon two miR-122-binding sites near the $5^{\prime}$ end of the HCV genome, S1 and S2. Viral RNA with base substitutions in both $\mathrm{S} 1$ and $\mathrm{S} 2$ failed to produce infectious virus in transfected cells, and translation of HCV RNA was reduced over $50 \%$ by mutations in either S1 or S2. The authors concluded that miR-122 promotes replication by binding directly to both sites in the genomic RNA and, at least in part, by stimulating internal ribosome entry site (IRES)-mediated translation.

Multiple Diverse Circoviruses Infect Farm Animals and Are Commonly Found in Human and Chimpanzee Feces

Circoviruses, which have small, circular, single-stranded DNA genomes, infect birds, and porcine circoviruses infect pigs. Circovirus infections, which are thought to occur mainly through fecal-oral transmission, can cause a wide range of severe symptoms with significant economic impact. Li et al. (L. Li et al., J Virol 84:1674-1682, 2010, https://doi.org/10.1128/JVI.02109-09) used viral metagenomics to identify circoviruslike DNA sequences and characterized 15 circular viral DNA genomes in stool samples from humans in Pakistan, Nigeria, Tunisia, and the United States and from wild chimpanzees. Phylogenetic analysis indicated that some viral genomes were part of a previously unrecognized genus in the Circoviridae family that was tentatively named Cyclovirus. There was limited genetic overlap between cycloviruses in human stool samples and local cow, goat, sheep, camel, and chicken meat samples, indicating that the majority of the 25 Cyclovirus species identified might be human viruses. Thus, the genetic diversity of small, circular DNA viral genomes in various mammals, including humans, is significantly larger than was previously recognized.

\section{Mechanism of Neutralization by the Broadly Neutralizing HIV-1 Monoclonal} Antibody VRC01

The broadly neutralizing human immunodeficiency virus type 1 (HIV-1) monoclonal antibody VRC01 forms a complex with the HIV-1 gp120 core, which partially mimics the interaction of the primary virus receptor CD4 with gp120. Li et al. (Y. Li et al., J Virol 85:8954-8967, 2011, https://doi.org/10.1128/JVI.00754-11) extended the investigation of the VRC01-gp120 core interaction to the biologically relevant viral spike. Mutagenesis studies indicated that VRC01 contacts within the gp120 loop D, the CD4 binding
Citation American Society for Microbiology 2017. Classic Spotlight, 2010 and 2011: Articles of significant interest selected from the Journal of Virology archives by the editors. J Virol 91:e01433-17. https://doi.org/ 10.1128/JVI.01433-17.

Copyright $\odot 2017$ American Society for Microbiology. All Rights Reserved. 
loop, and the V5 region were necessary for optimal VRC01 neutralization, as suggested by the crystal structure. VRC01 interaction with the native viral spike did not occur in a CD4-like manner. Further, VRC01 did not show significant reactivity with human antigens, which is important for potential in vivo applications. VRC01 interacts with gp120 in the context of the functional spike in a manner distinct from that of CD4, by precisely targeting the CD4 binding sites without alterations of Env spike configuration.

Human and Rhesus APOBEC3D, APOBEC3F, APOBEC3G, and APOBEC3H

Demonstrate a Conserved Capacity To Restrict Vif-Deficient HIV-1

Human immunodeficiency virus type 1 (HIV-1) encodes a protein called viral infectivity factor (Vif) that fosters replication in $\mathrm{CD}^{+}{ }^{+} \mathrm{T}$ lymphocytes and promotes pathogenesis by triggering the degradation of the retrovirus restriction factor APOBEC $3 G$. Hultquist et al. (J. F. Hultquist et al., J Virol 85:11220-11234, 2011, https://doi.org/10 $.1128 / \mathrm{JVI} .05238-11$ ) reported a comprehensive analysis of the complete, seven-member human and rhesus APOBEC3 families in HIV-1 restriction. Three other human APOBEC3 proteins, APOBEC3D, APOBEC3F, and APOBEC $3 \mathrm{H}$, were found to be potent HIV-1 restriction factors. These four proteins, which are expressed in $\mathrm{CD}^{+}{ }^{+} \mathrm{T}$ lymphocytes, were able to mutate proviral DNA and can be counteracted by HIV-1 Vif. APOBEC3D, APOBEC3F, APOBEC3G, and APOBEC3H of the rhesus macaque also restrict Vif-deficient HIV-1, and they are all neutralized by the simian immunodeficiency virus Vif protein. In contrast, neither human nor rhesus APOBEC3A, APOBEC3B, or APOBEC3C had a significant impact on HIV-1 replication. These data implicate a combination of four APOBEC3 proteins-APOBEC3D, APOBEC3F, APOBEC3G, and APOBEC3H-in HIV-1 restriction. 